\title{
THERMAL AND MASER PHENOMENA \\ IN ROTATIONALLY EXCITED OH
}

\author{
PATRICK PALMER \\ University of Chicago, Chicago, Ill., U.S.A.
}

\begin{abstract}
Both maser emission and thermal emission and absorption have been observed from the rotationally excited ${ }^{2} \pi_{3 / 2}, J=5 / 2$ and ${ }^{2} \pi_{1 / 2}, J=1 / 2$ states of $\mathrm{OH}$. Conditions in the interstellar gas are discussed.

My colleagues (B. Zuckerman, Univ. of Maryland and Univ. of California at Berkeley, and L. J. Rickard, Univ. of Chicago) and I have been studying radiation from the rotationally excited ${ }^{2} \pi_{3 / 2}, J=5 / 2$ and ${ }^{2} \pi_{1 / 2}, J=1 / 2$ states of $\mathrm{OH}$. Both maser emission and thermal emission and absorption have been observed. Maser emission has been found in several new sources, including NGC 7538, M17, and OH69-1, and time variations in the previously known sources have been monitored. In the later study, variations with as rapid an $e$-folding time as $8 \mathrm{~h}$ have been seen.

The thermal emission feature in the ${ }^{2} \pi_{1 / 2}, J=1 / 2$ state in Sgr B2 (discovered by Gardner and Ribes) was re-observed and the corresponding features in the ${ }^{2} \pi_{3 / 2}$, $J=5 / 2$ state were detected. In W3, however, absorption was found in the ${ }^{2} \pi_{3 / 2}, J=5 / 2$ state. This is the shortest-lived state from which absorption is seen in the interstellar medium at any wavelength, radio or optical. The short lifetime of the state suggests very high densities for its excitation. Such densities in an extended source have previously been suggested only for the densest of the mm-wavelength like sources.
\end{abstract}

Full details of these studies will appear in the Astrophysical Journal.

\section{Patrick Palmer}

Department of Astronomy and Astrophysics,

Ryerson Laboratory 162,

1100-14 East 58th Street,

Chicago, Ill. 60637, U.S.A.

\section{DISCUSSION}

Wynn-Williams: New Caltech measurements by Werner, Wilson and myself of the position of the 1720 $\mathrm{MHz} \mathrm{OH}$ maser in W3 (continuum) indicate that it does not coincide with either an $\mathrm{H}_{2} \mathrm{O}$, an IR or radio component, and therefore may not be so easily related to the $5-\mathrm{cm} \mathrm{OH}$ absorptions.

Townes: What is the linear size and the total mass of this dense region?

Palmer: Placing it at the edge of the W3 complex, its linear size is $0.3 \mathrm{pc}$. At a density of $10^{6} \mathrm{~cm}^{-3}$, the mass is a few hundred solar masses. 